\title{
LÓGICAS DE LA PRODUCCIÓN DE INVESTIGACIÓN EN EL CAMPO DEL ARTE ${ }^{1}$ RESEARCH PRODUCTION LOGICS IN THE FIELD OF ART
}

\author{
CLARA AZARETTO \\ clara.azaretto@gmail.com \\ Facultad de Bellas Artes. Universidad Nacional de La Plata. Argentina
}

Recibido 21/3/2018 | Aceptado 11/7/2018

\section{Resumen}

El presente proyecto aborda el tema de la investigación en arte a partir del estudio de proyectos de investigación que se desarrollaron en la Facultad de Bellas Artes (FBA) de la Universidad Nacional de La Plata (UNLP). Se pondrá foco en las estrategias metodológicas que adoptaron los investigadores para abordar sus objetos de estudio. Nos referimos a los recortes temáticos que privilegian, cómo expresan sus problemas, qué tipo de hipótesis formulan, qué tipos de diseños predominan y cuáles son sus propuestas en relación con el análisis de los datos.

\section{Palabras clave}

Investigación; arte; metodología

\begin{abstract}
His project deals with the subject of art research from the study of research projects that were developed in the Facultad de Bellas Artes (FBA) de la Universidad Nacional de La Plata (UNLP). It will focus on the methodological strategies adopted by researchers to address their objects of study, we refer to: thematic cuts that privilege how they express their problems, what kind of hypothesis they formulate, what types of designs predominate, what are their proposals in relation with the analysis of the data.
\end{abstract}

\section{Keywords}

Investigation; art; methodology

Este proyecto pertenece al Programa de Incentivos a los docentes investigadores, Ministerio de Educación de Presidencia de la Nación. Código del proyecto: 11/B338. Período acreditado desde el 01/01/2018 hasta el 31/12/2019. 


\section{DIRECTORA}

Azaretto, Clara

\section{CODIRECTORA}

Valesini, Silvina

\section{INTEGRANTES}

Paduán, Fernando

Quiroga, Jorgelina

Quiroga, Pablo

Wood, Lucía

\section{Objetivo del proyecto}

Analizar el escenario de la Investigación en arte en la Facultad de Bellas Artes (FBA) de la Universidad Nacional de La Plata (UNLP) en función de reconocer las lógicas subyacentes a los procesos de investigación desarrollados y en desarrollo.

\section{Marco teórico}

Entendemos la metodología de la investigación como una disciplina reconstructiva, cuyo objeto es identificar y hacer explícitas las regularidades del proceder en la práctica de la investigación científica. Desde nuestro posicionamiento, consideramos la ciencia como un modo particular de producción de conocimiento, en el que encontramos como rasgo distintivo la búsqueda de un criterio externo, es decir, la contrastabilidad empírica como criterio de validación de las construcciones teóricas — diferenciándose así de otros modos o métodos de producción de conocimiento- (Samaja, 2003a). En la investigación en arte, como en toda práctica investigativa, encontramos la tensión propia del método de la ciencia, entre la búsqueda de descubrimiento de un nuevo conocimiento y las exigencias de validación.

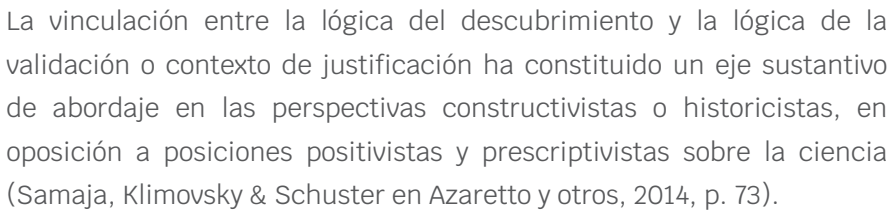


Reconocer ambos contextos, el de descubrimiento y el de validación, como lógicas inseparables e inherentes al método de la ciencia, nos convoca a su vez a pensar el conocimiento científico desde esa misma dialéctica. Con este sentido, nos serviremos de la lectura metodológica de las producciones investigativas para analizar la concepción y la dialéctica de ambos polos: descubrimiento y validación.

La investigación en el campo del arte cuenta con una reconocida trayectoria en los espacios académicos, aunque no existe coincidencia sobre la misma. Sigue vigente la polémica acerca de su estatuto. El debate se establece entre quienes sostienen la idea de que la investigación en arte se reduce a la explicitación de los procesos de producción de la obra, y quienes acuerdan en entenderla como otra forma de elaboración de conocimiento con criterios de validación diferentes a los del arte. En la primera de estas posiciones el artista y el investigador se identifican, mientras que en la segunda se establece una distancia entre ellos. Otra polarización se establece a la hora de pensar las relaciones del arte con la ciencia. En este caso no se pone en cuestión el qué se entiende por ciencia, sino más bien se sostiene la ilusión de una respuesta unívoca acerca de esta. Apresuradamente se la homologa a la concepción positivista, sin considerar que esta es una, entre otras, versiones de la ciencia.

A modo de aproximación a la resolución del conflicto resulta pertinente examinar críticamente -en el sentido kantiano del término- los procesos de producción que protagonizan los investigadores en el campo de las artes. El análisis de los mismos nos permitirá inferir las concepciones de ciencia y arte que subyacen a la práctica investigativa en el campo, así como los modos que caracterizan su producción, y tomar posición al respecto.

Roberto Fajardo-González plantea las coordenadas para pensar el estatuto de la investigación en arte. En uno de sus artículos (s/d), señala los polos en los cuales se desarrolla la investigación en el área, marcando su relación con las posiciones y preguntas que acompañan el proceso de inserción del arte en los espacios académicos, específicamente, en aquellos cuya propuesta no se centra exclusivamente en los aspectos teóricos del Arte.

Los debates en relación con la indagación artística en el contexto de la educación superior, de acuerdo con Henk Borgdorff (2006), nos permiten enmarcar nuestra tarea, ya que contemplan el perfil de quien es a su vez artista, docente e investigador. Al respecto, el autor postula una distinción interesante: "Voy a distinguir entre (a) investigación sobre las artes, (b) investigación para las artes, e (c) investigación en las artes» (Borgdorff, 2006, p. 8). 
Define a la primera como aquella que construye como objeto de estudio la práctica artística entendida ampliamente. Reconoce en este grupo las investigaciones que buscan arribar a conclusiones válidas sobre la práctica artística pero constituyéndose desde una distancia teórica. Por su parte, identifica al segundo tipo, las que denomina para las artes, como aquella que describe de modo específico la investigación aplicada. En ella «el arte no es tanto el objeto de investigación, sino su objetivo» (Borgdorff, 2006, p. 9). Los trabajos que integran este grupo, deberán aportar descubrimientos e instrumentos que contribuyan a forjar prácticas concretas de diferentes maneras. Por último en esta tricotomía, aparece la investigación en las artes, que para el autor «es el más controvertido de los tres tipos ideales de investigación» (Borgdorff, 2006, p. 9). La misma no asume la separación entre sujeto y objeto, es decir, no considera que exista distancia entre el investigador y la práctica artística, ya que se la supone parte esencial tanto del proceso de investigación como de sus resultados.

Este acercamiento entre sujeto y objeto está basado en la idea de que no existe ninguna separación fundamental entre teoría y práctica en las artes. Después de todo, no hay prácticas artísticas que no estén saturadas de experiencias, historias y creencias; y a la inversa, no hay un acceso teórico o interpretación de la práctica artística que no determine parcialmente esa práctica, tanto en su proceso como en su resultado final. Conceptos y teorías, experiencias y convicciones, están entrelazados con las prácticas artísticas y, en parte por esta razón, el arte es siempre reflexivo. De ahí que la investigación en las artes trate de articular parte de este conocimiento expresado a través del proceso creativo y en el objeto artístico mismo.

El debate sobre la investigación artística, según Borgdorff (2006), ha demostrado que resulta provechosa la utilización de nuevas distinciones, que no deriven directamente de elementos del lenguaje artístico en particular, sino que observen el objeto, el proceso y su contexto. Este autor comprende por objeto lo que representa la obra de arte —es decir, la composición, la imagen, la actuación, el diseño-, y la estructura dramática, el argumento, la disposición del escenario, el material, la música. Por proceso entiende la producción de arte: creación, producción, ensayo, desarrollo de imágenes y conceptos o pruebas. En tanto que el contexto representa lo que el autor retoma como el mundo del arte, que comprende también la recepción del público, el entorno cultural e histórico, la industria cultural, etcétera. Compartimos con Borgdorff:

La crítica a diferentes clasificaciones con respecto a las Investigaciones artísticas que se intentaron realizar, y la hacemos extensiva a las 
clasificaciones de investigaciones en otros campos disciplinares. En primer lugar estas clasificaciones no cumplen con los requisitos necesarios de toda clasificación: clases exhaustivas y mutuamente excluyentes. ${ }^{2}$ En segundo lugar, las clasificaciones previas pueden producir efectos paralizantes para quien se inicia o aborda una investigación en el área, pues tienen una connotación prescriptiva: toda investigación en el campo del arte debe entrar en alguna de estas categorías clasificatorias (Azaretto, 2017, p. 20).

En este sentido es importante diferenciar el trabajo de producción artística y el trabajo de investigación académica o científica, de igual manera que en otras disciplinas distinguimos a la investigación científica de las intervenciones profesionales, no en cuanto lo estrictamente procedimental sino en lo que hace a sus objetivos. Sostenemos que estos aspectos no están claramente diferenciados en los trabajos sobre la temática. "Además es importante señalar que no es condición necesaria ni suficiente cursar una carrera en Arte para producir obras categorizadas como artísticas; ni es obligación de los artistas producir investigaciones en el área» (Azaretto, 2017, p. 20).

Algunos autores, como Gregorio Klimovsky (1994), consideran que no es posible hablar de un único método de investigación científica. Sostienen que aludir a un método o a "el método» implica referirse en realidad a un vasto conjunto de tácticas empleadas para producir el conocimiento (Klimovsky, 1994). A nuestro entender, analizan el tema en un solo plano y consideran el método unidimensionalmente homologándolo con técnicas. Confunden el método con las metódicas particulares. Este punto es crucial en relación con el comprender lo que se juega en la denominación: la Investigación en Arte y su especificidad (Azaretto, 2017, p. 22).

Nuestro proyecto cuenta con antecedentes en investigaciones realizadas en otros campos disciplinarios, como el de la Arquitectura y el del Psicoanálisis, donde se plantean problemáticas análogas a las que se formulan en relación con el debate sobre La investigación en arte. $^{3}$

\footnotetext{
2 Por exhaustividad y clases mutuamente excluyentes: toda investigación artística queda incluida en una y sólo una de estas clases propuestas.

${ }^{3}$ Para ampliar en esta temática se puede consultar Investigar en Psicoanálisis (Azaretto y otros, 2014).
} 


\section{Problema de investigación}

A continuación, mencionaremos algunos de nuestros interrogantes iniciales: ¿cómo se expresa la producción de conocimiento científico en el campo del arte? ¿Qué modalidades adopta la lógica de descubrimiento y validación? ¿Son reconocibles condiciones metodológicas específicas dentro de la investigación en arte? Las diferencias existentes ¿pueden incluirse dentro de cánones consensuados de cientificidad? ¿Están asociadas a operaciones y a procedimientos metodológicos específicos - tipo de estudio y diseño, fuentes, estrategias y técnicas instrumentales y/o de análisis de datos-? ¿O se producen rupturas? En ese caso, ¿en qué aspectos? ¿Cómo describen los investigadores en arte la lógica propia de sus procesos de investigación?

\section{Hipótesis}

Una descripción sistemática de los modos en que los investigadores en arte conciben su objeto de estudio, por encuadrarse en aquella producción que participa de las condiciones institucionales académicas actuales, aportará elementos para reconocer estilos que adopta la producción científica en el tema.

Para abordar el interrogante sobre la especificidad de la investigación en el campo del arte debe distinguirse el método de investigación, en tanto condición de posibilidad misma del acto de investigar, de las configuraciones singulares que asume este método en cada disciplina de estudio (Samaja, 1993). Los tipos de esquemas y diseños de investigación, los modelos teóricos de base, los modos de construcción y análisis de datos, los modos de esquematización del objeto de estudio, entre otros, constituyen los rasgos que definen la especificidad del campo de investigación de una disciplina.

En línea con lo planteado, creemos que las formas de producción académica en Arte se corresponden con los Métodos Generales de las formas de la cientificidad. Las investigaciones en arte, como en otras disciplinas, tienen como propósito último el descubrimiento de nuevo conocimiento y aspiran a validarlo; llevan adelante acciones que marcan la lógica de un proceso investigativo orientado a problematizar un fenómeno y/o constructo teórico; suponen un esquema de investigación y diseñan estrategias para abordar el problema, analizarlo e interpretarlo. Asimismo, comparten con las formas de la cientificidad el requisito de ser comunicables y de dar cuenta de su lógica de producción. Están sujetas, igual que las investigaciones en otros campos disciplinares, a condiciones de coherencia interna. 
En todo caso, la especificidad de la investigación en arte radica en las metódicas o en la elección de procedimientos para llevarlas a cabo, vinculadas con las particularidades de la producción artística y sus fundamentos.

\section{Metodología}

Esta investigación se encuadra en un diseño de tipo descriptivointerpretativo. Se utilizará como criterio muestral el de saturación, aplicado al muestreo teórico (Strauss \& Corbin, 2002). Se estudiarán los proyectos y los productos de investigación ${ }^{4}$ que han tenido curso en los últimos años, ${ }^{5}$ como también las tesis de doctorado y de maestría, tanto en curso como terminadas en el período a definir.

De este modo, la población de estudio estará conformada por equipos de docentes-investigadores y tesistas — de grado, maestría o doctoradopertenecientes al ámbito académico de la FBA. Se incluirán al menos diez casos de cada grupo; ${ }^{6}$ así como proyectos y resultados de investigación -a través de informes, artículos, ponencias, de divulgación de resultados- de los mismos equipos y tesistas. Se intentará reflejar en dicha selección, la diversidad de disciplinas y áreas de investigación en el campo.

Por último, las categorías de análisis a emplear se determinarán en torno al aspecto a investigar, es decir, si se trata del abordaje de los proyectos de investigación o si nos abocamos al examen de los modos en que investigadores y tesistas en arte piensan sus objetos de estudio. En el primer caso se analizarán: ejes temáticos propuestos, campos de problematización, delimitación teórica y empírica del objeto de estudio, tipos de hipótesis, fuentes utilizadas, procedimientos operacionales en juego en la delimitación del objeto de estudio, estrategias instrumentales, procedimientos operacionales en el análisis de los datos, lógica del descubrimiento y lógica de la validación, exposición de resultados —enunciación/argumentación/enunciado-.

En el segundo caso, se indagará sobre cómo conciben los campos de problematización en arte, la delimitación del objeto de

\footnotetext{
${ }^{4}$ En distintos formatos, como proyectos y materiales de divulgación de resultados y otros.

${ }^{5}$ La delimitación temporal se definirá al hacer un primer acercamiento exploratorio a la problemática y la consulta con informantes clave, permitiendo identificar un criterio que no resulte arbitrario.

${ }^{6}$ Se propone abordar tanto los proyectos de investigación, como entrevistar a algunos de los directores/tesistas.
} 
estudio, los procedimientos operacionales en dicha delimitación, los procedimientos operacionales utilizados en el análisis de los datos, los principales obstáculos reconocidos durante el proceso de investigación, la concepción de ciencia y la metodología de la investigación en la que se sustentan, la relación entre la posición del artista y la del investigador en arte.

\section{Referencias}

Azaretto, C. (Coord.). (2017). Investigar en Arte. La Plata, Argentina: EDULP. Recuperado de http://sedici.unlp.edu.ar/bitstream/handle/10915/64154/ Documento_completo.pdf-PDFA.pdf?sequence=1

Azaretto, C.; Ros, C.; Barreiro Aguirre, C.; Murillo, M.; Wood, L.; Estévez, A.; Messina, D. (2014). Investigar en Psicoanálisis. Buenos Aires, Argentina: JCE Ediciones.

Borgdorff, H. (2006). El debate sobre la investigación en las artes. Recuperado de https://es.scribd.com/document/279835961/ BORGDORFF-El-Debate-Sobre-La-Investigacion-en-Las-Artes Fajardo-González, R. (s/d). La investigación en el campo de las Artes Visuales y el ámbito académico universitario. Recuperado de http://www. google.co.cr/\#q=La+investigación+en+el+-campo+de+las+artes+visuales Klimovsky, G. (1994). Las desventuras del conocimiento científico. Buenos Aires, Argentina: Eudeba.

Samaja, J. (1993). Epistemología y metodología. Buenos Aires, Argentina: Eudeba.

Samaja, J. (2003a). Semiótica de la ciencia. Los caminos del conocimiento. Inédito.

Samaja, J. (2003b). Semiótica de la ciencia. El papel de las hipótesis y de las formas de inferencia en el trabajo científico. Inédito.

Strauss, A y Corbin, J. (2002). Bases de la investigación cualitativa. Técnicas y procedimientos para desarrollar la teoría fundamentada. Antioquia, Colombia: Editorial de la Universidad de Antioquia. Recuperado de https:// diversidadlocal.files.wordpress.com/2012/09/bases-investigacioncualitativa.pdf 